\title{
La politique "ALARA" d'Électricité de France*
}

\author{
L. STRICKER**, R. DOLLO**
}

(Manuscrit reçu le 16 décembre 1994, accepté le 19 janvier 1995)

RÉSUMÉ En 1992, Électricité de France (EDF) a décidé de mieux intégrer la radioprotection dans la gestion globale de l'entreprise et s'est fixé pour objectif d'assurer la même-protection pour les travailleurs des entreprises extérieures que pour ceux d'EDF. Cette décision est intervenue dans un contexte marqué par la dégradation des résultats dosimétriques sur les sites français et par la publication des nouvelles recommandations de la CIPR. Cet exposé décrit la politique retenue par EDF, tant au niveau national qu'au niveau local, pour atteindre ces objectifs. Elle consiste à mettre en place des organisations légères et incitatives, à motiver tous les acteurs, à développer et à faire utiliser des outils appropriés. Les premiers résultats positifs de la politique ALARA sont ensuite exposés avec des exemples concrets et une analyse de l'évolution des indicateurs globaux.

ABSTRACT In 1992, Electricité de France (EDF) decided to improve the degree to which radiological protection was incorporated in the overall management of the utility and set itself the objective of ensuring the same level of protection both for contractors' and EDF's workers. This decision was taken in a context marked by a deterioration in exposure values for French plants and by the new ICRP recommendations. This document describes the policy adopted by EDF at both the corporate and plant levels to meet these objectives, by setting up management systems responsive but not cumbersome, a broad policy of motivation, the development and use of suitable tools. The first positive results of EDF's ALARA policy are stated together with concrete examples and analysis of the changes in global indicators.

\section{Introduction}

Au 31 décembre 1993, le parc nucléaire d'EDF comportait 56 tranches nucléaires en exploitation, dont 54 réacteurs à eau sous pression (REP), correspondant à une puissance nette installée de 58880 MW. En 1993, le parc nucléaire a produit 350,2 TWh sur une production totale d'électricité de 450,6 TWh. La production nucléaire a augmenté de $8,8 \%$ par rapport à celle de 1992. Elle représente désormais $78 \%$ de l'électricité produite en France.

* Communication présentée lors des Journées SFRP, "L'optimisation de la radioprotection des travailleurs dans les domaines électronucléaire, industriel et médical", La Rochelle, 2021 septembre 1994.

** Électricité de France, Exploitation du Parc Nucléaire (EDF/EPN), 92060 Paris La Défense cedex 57, France. 
La première démarche ALARA à EDF a été appliquée lors du remplacement des générateurs de vapeur de Dampierre 1, pendant sa phase de préparation en 1988-1989 et sa phase de réalisation en 1990.

En France, à cette époque, c'est-à-dire vers la fin des années 80, ALARA ne faisait pas explicitement partie de la culture de radioprotection. Ce principe n'est d'ailleurs apparu dans la législation française pour les installations nucléaires qu'en 1988 [2]. Durant cette même période, on observe également une dégradation progressive des résultats dosimétriques sur les sites français, tant en valeur absolue que relativement aux résultats des autres pays (Fig. 1).

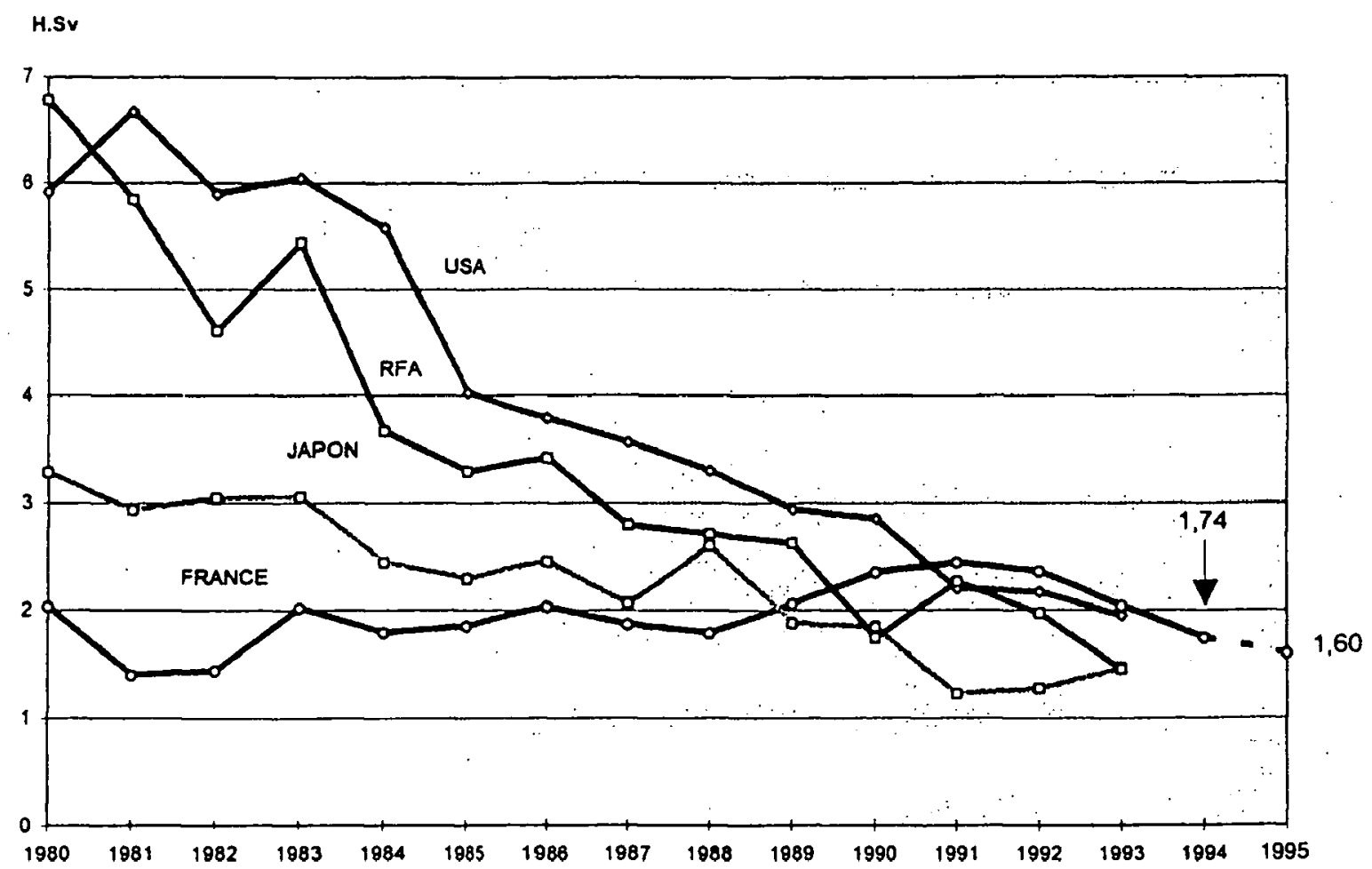

Fig. 1 - Dose collective moyenne annuelle par réacteur (Allemagne, France, Japon, RFA, USA).

Mean annual collective doses from PWRs in various countries.

Les résultats obtenus sur le remplacement des trois générateurs de vapeur de Dampierre, $2,13 \mathrm{~h} . \mathrm{Sv}$, soit $0,7 \mathrm{~h} . \mathrm{Sv}$ par générateur de vapeur remplacé (Fig. 2), en 70 jours - meilleure performance mondiale à l'époque [4] - ont clairement démontré à EDF qu'il était possible, par l'application de la démarche ALARA, de passer efficacement d'une "radioprotection de contrôle a posteriori du respect des limites de doses" à une gestion a priori des expositions individuelles et collectives. 


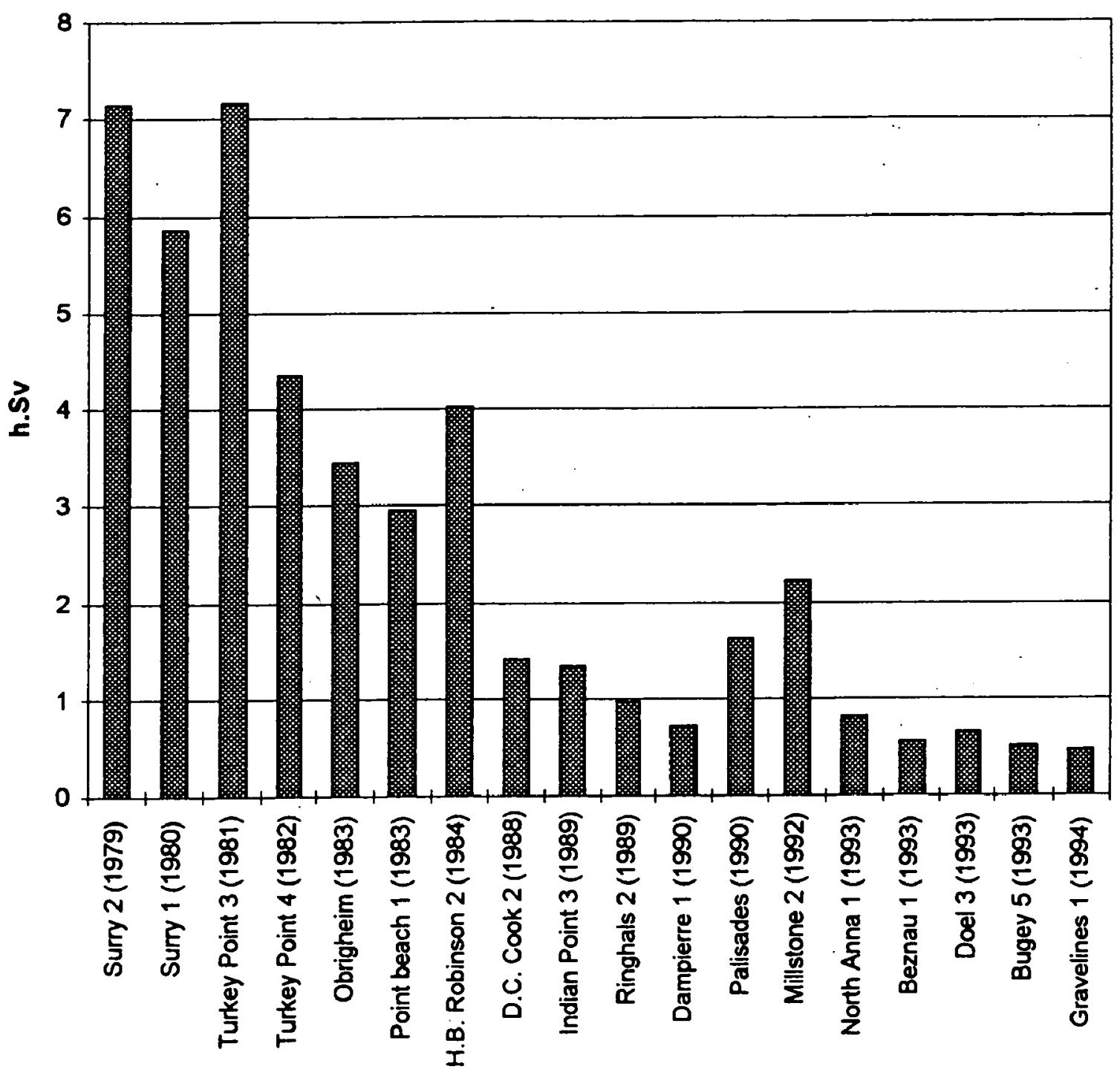

Fig. 2 - Remplacement des générateurs de vapeur - dose collective par générateur de vapeur (par ordre chronologique).

Replacement of steam generator (SG) - collective dose per SG (in chronological order).

Dans ce contexte, désireuse de généraliser la démarche à l'ensemble des opérations effectuées sur le parc français, EDF s'est fixé, en 1992, un double objectif ambitieux de réduction des expositions, anticipant de fait la diminution des limites maximales admissibles des doses individuelles :

- mieux intégrer la radioprotection dans le management global de l'entreprise et passer de 2,44 h.Sv/tranche en moyenne en 1991 à $1,6 \mathrm{~h} . \mathrm{Sv}$ en 1995 ;

- assurer la même protection pour les travailleurs des entreprises extérieures que pour ceux d'EDF et, en priorité, réduire les expositions des groupes de travailleurs ayant les niveaux de dose individuels les plus élevés. 
Pour atteindre ces objectifs, EDF :

- a créé des comités et des groupes ALARA spécifiques ;

- insuffle une culture de radioprotection dans l'entreprise ;

- a mis en place une politique de motivation de tous les acteurs concernés par les expositions aux rayonnements ionisants ;

- développe et utilise des outils ALARA.

\section{Un engagement explicite de la hiérarchie}

La direction d'EDF a clairement exprimé, à de multiples reprises, les objectifs précédents et rappelé que la réduction des expositions faisait partie de ses priorités. Récemment, cet engagement a été repris dans un document [1] d'une quarantaine de pages, sorte de loi-cadre décrivant les orientations et les objectifs de la radioprotection de l'entreprise. Chaque objectif fait l'objet d'un plan d'actions devant aboutir d'ici l'an 2000. Ce document, préfacé par le directeur général adjoint d'EDF, a été très largement diffusé dans l'entreprise ainsi qu'à l'extérieur.

\section{Une organisation légère et incitative}

EDF exploite 54 REP sur 17 sites répartis sur tout le territoire national. Les comités et les groupes ALARA mis en place, qui restent légers et essentiellement incitatifs, se situent donc tout naturellement à deux niveaux : national et local (Fig. 3)

\subsection{Niveau national}

\section{a) Un comité ALARA national}

Ce comité a été créé en décembre 1991; il est dirigé par un membre du Comité de direction de la branche "Production d'électricité nucléaire" d'EDF, et s'appuie sur le département "Sécurité-radioprotection-environnement". Il comporte deux types de membres : les membres permanents, responsables de services ou de départements nationaux (radioprotection, développement d'outillages, conception des installations futures), des représentants de la hiérarchie des centrales nucléaires, qui changent par tiers tous les 18 mois afin que tous les sites soient représentés à tour de rôle dans ce comité.

Ce comité définit les grandes lignes de la politique ALARA d'EDF, il entérine des doses cibles au niveau national par type de réacteur et par année en vue d'atteindre l'objectif de 1,6 h.Sv par réacteur en moyenne en 1995, il suscite une émulation entre les sites pour la réalisation de cet objectif, il suit des indicateurs de résultats, et favorise le retour d'expérience entre les sites. 


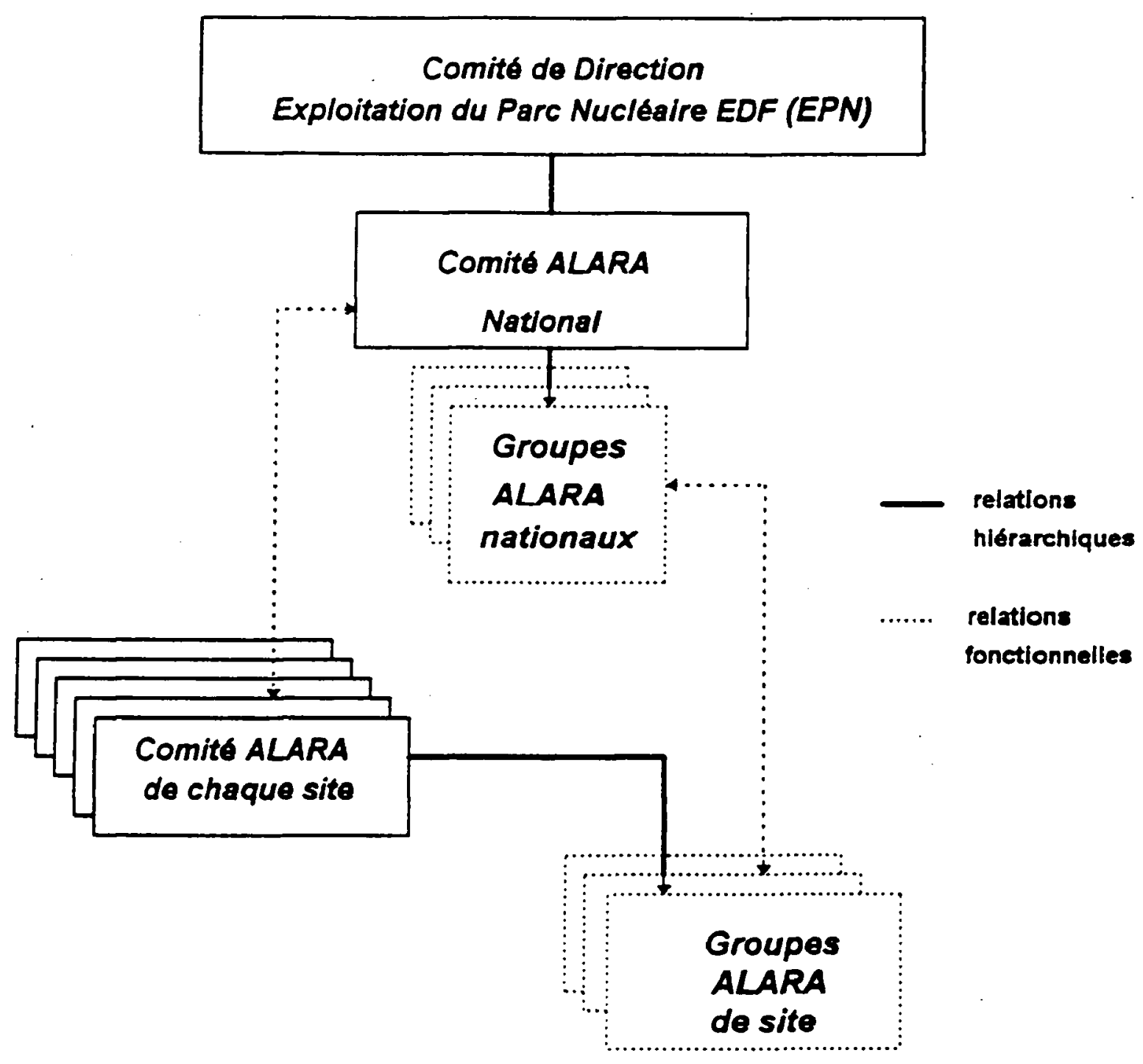

Fig. 3 - Organisation ALARA à EDF.

ALARA organisation at EDF.

b) Des groupes ou des missions ALARA ad hoc

De tels groupes peuvent être créés à l'occasion de problèmes nouveaux d'ampleur nationale ayant une incidence significative sur les expositions. Ainsi, en 1992, peu de temps après la découverte des fissures sur les couvercles de cuve et vue l'importance des expositions engagées pour contrôler, prévenir ou réparer ces fissures, un groupe ALARA national a été chargé, à la demande du comité ALARA national, de mettre en œuvre un programme ALARA adapté au problème. Ce groupe comporte non seulement des représentants des sites et des services centraux d'EDF mais aussi des principales entreprises concernées (Framatome, Jeumont Schneider ...). Un groupe du même type fonctionne depuis 1989 pour les remplacements des générateurs de vapeur (Dampierre, puis Bugey et Gravelines). 


\subsection{Niveau local}

Les organisations locales, créées à l'initiative des sites, apparaissent avant tout comme des organisations de coordination entre les divers métiers exercés sur les sites. Elles jouent aussi le rôle de relais : relais de la politique nationale vers les sites et les entreprises, relais des sites entre eux pour faciliter le retour d'expérience, relais des expériences de terrain et des propositions des sites vers les instances nationales.

Pratiquement, tous les sites ont créé un comité ALARA de site entre 1992 et 1993. Ce comité est dirigé par le directeur ou le directeur adjoint du site, ayant pouvoir décisionnel. Il est un lieu de dialogue. Il regroupe des représentants de tous les services (maintenance, planning, chimie, exploitation, services généraux, médecin du travail, ... et bien entendu radioprotection) et des représentants des entreprises extérieures.

A partir des objectifs dosimétriques propres au site, ces comités sélectionnent les chantiers pénalisants en dose qui seront particulièrement suivis et analysés, et décident des moyens à mettre en œuvre. La plupart des comités se font aider par des groupes pluridisciplinaires ou transverses qui sont chargés de leur proposer des actions efficaces pour réduire les expositions tant en agissant sur les débits (modalités de mise en arrêt, oxygénation,... développement de protections biologiques, ...) que sur les temps d'exposition (organisation des travaux d'échafaudage, de calorifugeage,... formation des intervenants, développement d'outillages spécifiques, ...).

\section{Une large politique de motivation de tous les acteurs}

\subsection{Convaincre les décideurs}

Plusieurs sites et services nationaux ont organisé, avec le concours d'experts, une journée destinée aux managers pour présenter et discuter le principe et la démarche ALARA et leur mise en œuvre à EDF. Le même type de journée a été organisée au niveau national pour sensibiliser les chefs d'entreprises extérieures nationales et discuter des implications de la politique ALARA d'EDF sur les relations entre exploitant et entreprises.

\subsection{Intégrer des objectifs dosimétriques dans les contrats de gestion}

La mise en place de cette politique s'est d'abord traduite par la détermination d'objectifs dosimétriques adaptés à chaque réacteur en fonction de ses caractéristiques propres (type de réacteur, existence de points chauds...) et des travaux à effectuer (visite partielle, visite décennale...). Ces objectifs, qui doivent rester cohérents avec la politique (doses-cibles) définie par le comité ALARA national, sont négociés entre les sites et l'état major national, puis intégrés dans les contrats de gestion annuels et les plans stratégiques triannuels sur lesquels la hiérarchie des sites s'engage vis-à-vis de la direction nationale. 


\subsection{Intégrer ALARA dans la culture de radioprotection}

En second lieu, EDF a engagé un vaste programme de sensibilisation et de formation de tous les acteurs : il s'agit là d'une première étape en vue d'intégrer ALARA dans la culture de l'ensemble des acteurs du domaine électronucléaire.

\section{a) Viser en priorité les acteurs des arrêts de tranche}

Les expositions pendant les arrêts de tranche représentent $80 \%$ de la dose annuelle. Aussi, la plupart des sites ont-ils organisé, avec le concours d'experts extérieurs, des sessions de formation de deux jours destinées aux équipes de préparation et de gestion des arrêts de tranche (chef d'arrêt et représentants des divers services concernés). Ces formations allient des présentations théoriques sur les fondements de l'optimisation de la radioprotection et des études pratiques d'expériences déjà réalisées sur d'autres sites.

\section{b) Former des formateurs}

Environ 40 agents EDF ont pris part durant une semaine à une formation d'animateurs-formateurs ALARA, destinée à démultiplier la formation ALARA sur les unités. Une nouvelle action de formation a été définie pour répondre aux attentes exprimées par le personnel d'encadrement des entreprises extérieures. Le but visé étant de mieux travailler ensemble, cette formation rendra ce personnel plus apte à s'approprier les objectifs du parc nucléaire dans le domaine de la maintenance, et à devenir acteurs dans la démarche ALARA.

\section{c) Intégrer ALARA dans les métiers}

L'efficacité des actions précédentes est relative, compte-tenu de la population touchée. En effet, il convient également de former les dizaines de milliers d'agents EDF et d'entreprises qui interviennent en centrale. C'est pourquoi, un module ALARA a été intégré, dès 1992, dans les habilitations à la radioprotection exigées pour travailler en zone contrôlée. Des modules de même type doivent être systématiquement inclus dans les formations aux métiers, y compris pour ceux qui ne travaillent pas en zone contrôlée mais dont le travail interagit sur les expositions professionnelles. Il s'agit, en particulier, des préparateurs des procédures de chantier ou des responsables de la conduite des réacteurs.

\subsection{Adapter des politiques d'information et de sensibilisation adaptées à chaque site}

Dans ce domaine, chaque site développe sa propre politique qui fait appel à l'imagination et à la créativité de ses agents : affichage des objectifs, journées d'informations sur ALARA à EDF et sur le site, affiches ALARA, vidéos, articles dans les journaux de site ou d'arrêt, challenge entre équipes, accueil de toutes les nouvelles équipes d'entreprises, inspections régulières des chantiers, 
points "radioprotection et ALARA" dans les réunions du comité d'hygiène et de sécurité ou dans les réunions d'arrêt de tranche...

Cette motivation de tous les acteurs apparaît particulièrement importante, tant dans la phase de préparation que pendant le déroulement des opérations pour réduire de nombreux dysfonctionnements qui occasionnaient en France jusqu'à $30 \%$ de la dose [6]. De plus, elle nécessite un effort permanent car le retour d'expérience de certains sites montre que, dès que la pression est allégée, les résultats se détériorent.

\subsection{Motiver les entreprises dans le cadre d'un partenariat contractuel}

La mise en oeuvre d'une politique ALARA nécessite la participation active des entreprises, puisque ce sont leurs agents qui effectuent la grande majorité des opérations de contrôle et de maintenance et qui reçoivent plus de $80 \%$ des expositions, et que leurs entreprises développent de nombreux process et outillages. EDF s'efforce donc, dans ce domaine comme dans d'autres, de développer une politique de partenariat contractuel, entre autres :

- en intégrant la réduction des expositions dans le cahier des charges des travaux à effectuer ;

- en étudiant le coût raisonnable des propositions des entreprises en matière d'organisation du travail, de modification de process ou de développement d'outillages ;

- en insérant des objectifs dosimétriques dans les commandes, sans pour autant donner de prime financière à la réalisation de ces objectifs ;

- en utilisant les doses effectivement engagées lors d'opérations antérieures de même nature comme critère de choix d'entreprises ;

- en réclamant des analyses de retour d'expérience en radioprotection en fin de chantier ;

- en favorisant la mise en place de programmes ALARA internes dans les entreprises.

Ces efforts communs figurent dans un protocole qui doit permettre à l'entreprise intervenante :

- de respecter strictement les normes européennes au niveau de la dosimétrie individuelle ;

- d'améliorer d'au moins $15 \%$ chaque année sa dosimétrie collective rapportée au nombre d'heures d'intervention en zone contrôlé ;

- d'obtenir une répartition plus équitable des doses entre ses intervenants : $80 \%$ d'entre eux ne devraient pas être éloignés de plus de $10 \%$ de la dosimétrie moyenne de la société. 
En fin d'année, les centres nucléaires de production d'électricité (CNPE) concernés et l'entreprise analysent ensemble l'évolution de la dosimétrie des intervenants, ainsi que la progression des comportements et des méthodes. L'évaluation annuelle des entreprises tient compte de la réduction de doses et de la qualité de la coopération instaurée entre les deux parties. Des manquements à ce protocole, en particulier sur la gestion de l'emploi des intervenants, pourraient conduire EDF à interrompre la collaboration avec les sociétés incriminées.

\section{Le développement et l'utilisation de moyens appropriés}

\subsection{Améliorer le suivi opérationnel des doses collectives et individuelles des agents EDF et d'entreprises par un système d'information}

Afin de mieux gérer de façon opérationnelle les expositions, EDF équipe progressivement ses sites d'un système informatique de gestion en temps réel des doses reçues lors de chaque séjour en zone contrôlée, voire lors de chaque passage dans une sous-zone. Par ailleurs, afin d'assurer la même protection à ses agents et à ceux des entreprises extérieures, EDF a mis en place une interconnexion informatique des différents sites (le système DOSINAT) qui permet de suivre nominativement les intervenants des entreprises lorsqu'ils changent de site. Cela permet donc d'intervenir au plus tôt, tant auprès des employeurs que par des investigations sur les conditions de travail dans les centrales, pour que l'optimisation de la radioprotection soit bien mise en œuvre dans le respect des limites de dose. DOSINAT a reçu l'autorisation de la Commission nationale "Informatique et libertés" (CNIL).

Dans les années à venir, cette base de données sera étendue aux autres maillons du cycle électronucléaire (COGEMA, CEA, Délégation générale à l'armement (DGA), car les intervenants des entreprises peuvent travailler successivement dans ces divers maillons du cycle. En collaboration avec le Groupe intersyndical de l'industrie nucléaire (GIIN), un système informatique baptisé DOSIMO a été défini : il permettra de gérer la dosimétrie opérationnelle des intervenants quel que soit leur lieu d'intervention.

De plus depuis la mi-1993, un nouveau carnet d'accès EDF est exigé de tout travailleur affecté à des travaux sous rayonnements lors de son arrivée sur un site. Ce carnet atteste la formation reçue par le travailleur et sa qualification professionnelle, il réunit les informations de suivi des doses reçues par le travailleur et fournit les résultats dosimétriques issus de DOSINAT et des contrôles anthropogammamétriques.

\subsection{Favoriser le retour d'expérience national et international}

La diffusion rapide du retour d'expérience étant un élément fondamental de la réussite de toute politique ALARA, EDF s'efforce d'accroître l'efficacité 
des échanges entre les sites. Ainsi, à côté des structures et des formations dont il a été question précédemment, à côté des bases de données propres à chaque site, des groupes de travail nationaux ont permis entre autres :

- de sélectionner les chantiers les plus coûteux en dose, d'attribuer chacun de ces chantiers à un site, de faire appliquer une procédure commune de prévision des doses du chantier, de recueillir des données pendant le chantier, de saisir ces données sous l'application informatique "DOSIANA", de faire rédiger un rapport de retour d'expérience et de les diffuser à l'ensemble des autres sites ; la première application de cette procédure a concerné l'ensemble des opérations liées aux contrôles et aux réparations des couvercles de cuve ; à terme, ce sont toutes les opérations d'arrêts de tranches qui devraient en bénéficier ;

- d'alimenter un classeur national de retour d'expérience sur les "bonnes pratiques" réductrices de dose, très largement diffusé sur les sites.

Parallèlement à cette volonté de faire fructifier son propre retour d'expérience, EDF a largement soutenu la mise en place du système ISOE [5] pour favoriser les échanges de retour d'expérience entre exploitants sur le plan international. Le président actuel d'ISOE est d'ailleurs M. Rollin du Comité de radioprotection d'EDF. EDF alimente régulièrement le système à partir de ses fiches de bonnes pratiques et des nouvelles opérations ou des problèmes rencontrés.

\subsection{Certifier les entreprises}

La qualité de la formation en matière de prévention des risques est essentielle. En 1990 a été créé le Comité français de certification des entreprises pour la formation et le suivi dosimétrique du personnel travaillant sous rayonnements ionisants (CEFRI). Cet organisme a été créé en plein accord avec les exploitants (EDF, COGEMA, CEA, DGA), le ministère de la santé, celui du travail et de l'industrie et les autorités en radioprotection. Il délivre des agréments après audit :

- aux organismes de formation ;

- aux entreprises de travail temporaire mettant à disposition du personnel ;

- aux entreprises employant du personnel travaillant dans les installations nucléaires.

L'agrément du CEFRI porte sur la qualité de la formation et sur l'organisation mise en place pour assurer le suivi dosimétrique et la surveillance médicale du personnel intervenant. A terme, seules les entreprises agréées par le CEFRI seront acceptées sur les sites nucléaires.

De plus, EDF modifie ses relations contractuelles avec les entreprises et introduit une clause qui spécifie que l'agrément de l'entreprise sera retiré si la rupture ou la suspension d'un contrat de travail entre cette entreprise et l'un de 
ses employés a pour objet l'atteinte ou le dépassement d'une limite réglementaire de dose.

\subsection{Une politique nationale d'outillages automatisés}

La mise en place d'une politique nationale de développement d'outillages automatisés s'avère être une piste particulièrement prometteuse. L'UTO (unité technique opérationnelle) qui est plus particulièrement chargée au sein de l'EDF de la maintenance, du développement et du suivi d'outillages spécialisés, a entrepris une réflexion sur la mise en oeuvre du concept ALARA et s'est dotée d'un comité ALARA.

\section{Premiers résultats}

\subsection{Un infléchissement de la tendance en 1992-1993 (Fig. 4)}

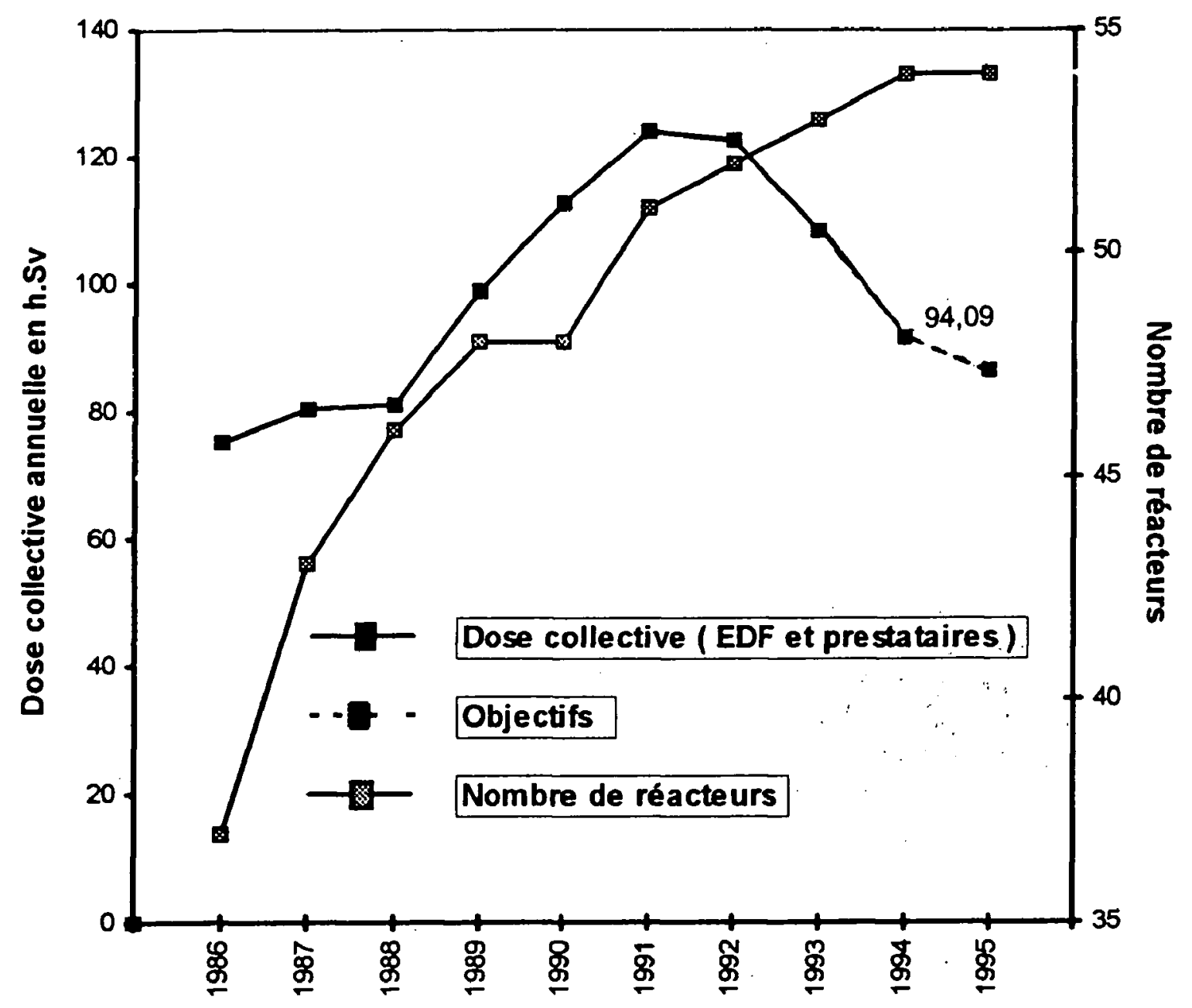

Fig. 4 - Dose collective annuelle et nombre de REP, 1986-1995.

Annual collective dose and number of PWRs, 1986-1995. 
Entre 1983 et 1989, la dose moyenne par tranche et par année fluctuait entre 1,8 et 2 h.Sv. En 1990, elle fut de 2,35 h.Sv et en 1991 de 2,44 h.Sv. La promotion d'ALARA à partir de 1992 a permis de commencer à inverser cette tendance : 2,36 h.Sv en 1992, 2,04 h.Sv en 1993 puis 1,74 h.Sv en 1994. L'objectif de 1,6 h.Sv en 1995 qu'EDF s'est donné reste donc très ambitieux.

\subsection{De grands chantiers optimisés}

Les opérations de remplacement des générateurs de vapeur (RGV) donnent systématiquement lieu à une préparation, un suivi et un retour d'expérience selon une démarche ALARA formalisée, dans le cadre d'un groupe de travail associant la Direction de l'équipement et l'exploitant. Le programme ALARA adopté pour le RGV s'articule essentiellement sur :

- une étude d'optimisation des actions de protection : niveaux d'eau dans les $\mathrm{GV}$, protections biologiques, décontamination des extrêmités des tuyauteries primaires ;

- une motivation forte des intervenants : formation et entraînement avant le RGV ; relations étroites entre équipe ALARA et intervenants pendant le chantier, en utilisant de nombreux moyens et supports (affichages, courbes dosimétriques du prévisionnel réalisé, réunions de chantier et de retour d'expérience, etc...).

Les résultats des RGV mettent en relief l'effet "ALARA".

\begin{tabular}{|ccc|}
\hline & RGV & Dose réalisée \\
\hline Dampierre 1 & $(1990)$ & $2,13 \mathrm{~h} . \mathrm{Sv}$ \\
\hline Bugey 5 & $(1993 / 1994)$ & $1,55 \mathrm{~h} . \mathrm{Sv}$ \\
\hdashline Gravelines & $(1994)$ & $1,41 \mathrm{~h} . \mathrm{Sv}$ \\
\hline
\end{tabular}

\subsection{Le cas des couvercles de cuve}

Le bilan dosimétrique des interventions sur les couvercles se présente comme suit à fin 1993 :

\begin{tabular}{|ccc|}
\hline & Valeur annuele & Valeur cumulé \\
\hline Dose 1991 & $1,9 \mathrm{~h} . \mathrm{Sv}$ & $1,9 \mathrm{~h} . \mathrm{Sv}$ \\
Dose 1992 & $9,0 \mathrm{~h} . \mathrm{Sv}$ & $10,9 \mathrm{~h} . \mathrm{Sv}$ \\
Dose 1993 & $7,0 \mathrm{~h} . \mathrm{Sv}$ & $17,9 \mathrm{~h} . \mathrm{Sv}$ \\
\hline
\end{tabular}


Une analyse faite sur les années 1991 et 1992 aboutissait à une prévision totale à fin 1992 de 16 h.Sv en l'absence de programme ALARA. Les 11 h.Sv atteints sur la même période attestent donc d'un gain de 5 h.Sv imputables à l'application de la méthode ALARA sur ces chantiers. L'opération de changement complet du couvercle sur Bugey a été réalisée début 1994 au coût de $0,2 \mathrm{~h} . \mathrm{Sv}$ pour 0,45 prévus. Cette prévision était très imparfaite, car s'appliquant au premier chantier de ce type. Néanmoins, le faible coût dosimétrique des aléas est encore à mettre au bénéfice de la bonne préparation ALARA du chantier, réalisée par le site en collaboration avec les services nationaux et les différentes entreprises extérieures.

\subsection{Les arrêts de tranches pour rechargement}

Golfech 1, réacteur de $1300 \mathrm{MW}$ mis en service en février 1991, a réalisé une performance dosimétrique de $0,47 \mathrm{~h}$.Sv sur l'année (dont $0,45 \mathrm{~h} . \mathrm{Sv}$ pour l'arrêt de tranche). Ce résultat est le meilleur enregistré à ce jour pour un réacteur français et montre que notre parc de réacteurs peut viser, en moyenne, une performance qui le fera approcher des meilleurs mondiaux.

\section{Conclusion}

En matière de radioprotection, l'objectif principal est bien évidemment la protection de l'homme qui passe en premier lieu par la surveillance des dose reçues. Mais, l'introduction d'ALARA dans la culture de l'entreprise modifie la philosophie de l'action et les comportements : il s'agit, en effet, pour protéger l'homme, de mettre aussi en œuvre une gestion du risque résiduel par :

- une approche prédictive, c'est-à-dire qui anticipe les expositions et les moyens de les réduire ;

- une approche efficace, c'est-à-dire raisonnable en ce qui concerne les moyens alloués à la protection ;

- une approche évolutive, c'est-à-dire qui tienne compte de l'évolution du contexte technique, économique et social.

Une telle gestion nécessite, pour réussir, la motivation de tous les acteurs concernés : le retour d'expérience montre qu'un tel consensus est d'autant plus aisément obtenu qu'il s'appuie sur une politique de transparence en ce qui concerne les risques résiduels dus aux rayonements ionisants et les moyens mis en oeuvre pour les réduire aussi bas que raisonnablement possible. 


\section{RÉFÉRENCES}

[1] EDF. - Livre blanc de la radioprotection. Paris : EDF, 1993.

[2] FRANCE. - Décret $\mathrm{n}^{\circ} 88-662$ du 6 mai 1988, relatif à la protection des travailleurs contre les dangers des rayonnements ionisants dans les installations nucléaire de base art.3. J.O.R.F., 8 mai 1988, p. 6719. In : Protection contre les rayonnements ionisants (Brochure $\mathrm{n}^{\circ}$ 1420). Paris : Journal officiel, 1990, vol. 2, 513-558.

[3] INTERNATIONAL COMMISSION ON RADIOLOGICAL PROTECTION (ICRP) Recommendations... (ICRP Publication 60), Ann. ICRP, 21 (1-3). Oxford : Pergamon press, 1991.

[4] JURION O., BLAIN A., LEFAURE C. - Gestion ALARA de la protection radiologique lors du remplacement des générateurs de vapeur de Dampierre 1 (France) en 1990. Radioprotection, 1992, 27 (2) 157-172.

[5] OCDE. AGENCE POUR L'ÉNERGIE NUCLÉAIRE (AEN). - Nuclear power plant occupational exposures in OECD countries 1969-1991. Paris : OECD, 1993.

[6] SCHIEBER C. - ALARA and work management. Radiation exposure management seminar, Pittsburgh, September 12-15, 1993. Pittsburgh : Westinghouse, 1993. 